\title{
Cigarette smoking and benign proliferative epithelial disorders of the breast in women: a case-control study
}

\author{
T E ROHAN, ${ }^{1 *} \mathrm{M} \mathrm{G} \mathrm{COOK},{ }^{2}+$ AND J A BARON ${ }^{3}$
}

From ${ }^{1}$ CSIRO Division of Human Nutrition, Kintore Avenue, Adelaide, South Australia 5000; ${ }^{2}$ Gribbles Pathology, 1 Goodwood Road, Wayville, South Australia 5034; ${ }^{3}$ Departments of Medicine and Community and Family Medicine, and Norris Cotton Cancer Center, Dartmouth-Hitchcock Medical Center, Hanover 03756, USA.

\section{ABSTRACT}

Study objective: The purpose of the study was to investigate the possible association between cigarette smoking and benign proliferative epithelial disorders of the breast.

Design: This was a case-control study with two different control groups.

Setting: The study was community based and took place in metropolitan Adelaide, South Australia. Subjects: 506 biopsy proven cases between 18 and 75 years were identified, of whom 39 could not be approached because of surgeon refusal, 66 would not be interviewed and 18 were untraceable, leaving 383 for inclusion in the study. Controls were 192 women who had had a negative breast biopsy, out of a possible 259 (17 surgeon refusals, 39 interview refusals, 11 untraceable); and a randomly selected group of women matched to the index group for age and area of residence (582 subjects were approached to enrol 383 controls).

Measurements and main results: All breast biopsies were examined by one pathologist and classified using a standard system. Sociodemographic and medical information was collected by trained interviewers using structured questionnaires. Overall, and within menopausal strata, risk of benign proliferative epithelial disease for women who had ever smoked and for current and ex-smokers was similar to that for women who had never smoked. In premenopausal women, using community? controls as the comparison group, risk decreased with cigarette-years of exposure, but the trend was not statistically significant. Risk appeared to increase when biopsy controls were used. There was no trend in the association when examined by degree of cytological atypia.

Conclusions: The data suggest that cigarette smoking is not related to the risk of benign proliferative epithelial breast disease.

Several features of benign breast disease suggest an aetiological role for the ovarian hormones. The disease is relatively common in premenopausal women, ${ }^{1}$ and its symptoms vary in severity with the menstrual cycle. ${ }^{2}$ Oestrogen replacement therapy is associated with increased risk of benign breast disease,$^{3-5}$ and use of oral contraceptives is associated with reduced risk. ${ }^{6}$ Additionally, some (but not all) studies have shown women with benign breast disease, when compared with controls, to have higher levels of

* Present address: NCIC Epidemiology Unit, Faculty of Medicine, McMurrich Building, University of Toronto, Toronto, Ontario M5S 1A8, Canada.

$\dagger$ Present address: Department of Histopathology, St George's Hospital, Blackshaw Road, London SW17, UK. oestradiol in their breast fluid, ${ }^{7}$ higher circulating levels of oestradiol, ${ }^{8-10}$ and lower circulating progesterone. ${ }^{10}$ This last observation suggests that benign breast disease is related to an imbalance between oestradiol and progesterone levels, and in particular to a relative excess of the former. ${ }^{10}$ If this is correct, factors which can alter the level of either or both of these hormones might be associated with altered risk of benign breast disease.

Cigarette smoking may be such a factor, since it has been associated with an early age at natural menopause, an increased risk of osteoporotic fractures, a reduced risk of endometrial cancer, and possibly decreased risk of uterine fibroids and endometriosis. ${ }^{11-13}$ Although oestradiol levels are similar in postmenopausal smokers and non- 
smokers, ${ }^{14}$ cigarette smoking has been associated with subtle disturbances in oestradiol metabolism. ${ }^{15}$ These observations suggest that an additional consequence of cigarette smoking might be a reduction in the risk of benign breast disease. To date, however, the results of studies of cigarette smoking and benign breast disease have been inconsistent. ${ }^{16-21}$ This may reflect, at least in part, the histological heterogeneity of benign breast disease. To address this limitation, we examined the association between cigarette smoking and benign breast disease in a case-control study of benign proliferative epithelial disorders of the breast.

\section{Methods}

The study has been described in detail elsewhere. ${ }^{22}$

\section{CASES AND CONTROLS}

Women were eligible for the study if they were aged between 18 and 75 years of age; if they resided in metropolitan Adelaide, South Australia; if they were registered on the state electoral roll (voter registration is compulsory); and if they did not have a history of breast cancer or biopsy confirmed benign breast disease. Potential cases had evidence of epithelial proliferation in biopsy material submitted to the major private pathology laboratory in Adelaide between January 1983 and October 1985. Three hundred and eighty three such cases were included in the study, 39 could not be approached due to refusal by the attending surgeon, 66 refused to be interviewed, and 18 were untraceable.

Two control groups were selected. One consisted of women whose first breast biopsy, submitted to the same laboratory during the same time period as that for the cases, did not show evidence of epithelial proliferation. Of the 259 women eligible for this control group (the "biopsy control group"), 192 were enrolled, 17 could not be approached due to surgeon refusal, 39 refused to be interviewed, and 11 were untraceable. The other control group was formed by matching to each case one subject selected at random from the electoral roll from those women similar in age to that of the case at diagnosis $(88 \%$ within \pm 1 year, the remainder with $\pm 2-3$ years) and living in the same suburb or a suburb similar to that of the case in terms of socioeconomic indicators. ${ }^{23} \mathrm{~A}$ total of 582 subjects was approached in order to enrol the 383 community controls. Reasons for non-participation were recorded during the latter stages of recruitment: of 51 nonparticipants, 30 refused and 21 were untraceable.

HISTOPATHOLOGICAL CLASSIFICATION OF BIOPSIES All breast biopsies were examined by one pathologist (M G C) who had no knowledge of the exposure histories of the subjects. Benign non-tumorous breast disease was categorised according to a system based on the methods of Bonser et al, ${ }^{24}$ Page et al, ${ }^{25}$ and Azzopardi. ${ }^{26}$ The group with epithelial proliferation included those with epitheliosis, radial scar, sclerosing adenosis, blunt duct adenosis, intraduct papilloma, fibrodenoma, fibroadenomatoid hyperplasia, and apocrine metaplasia. As described elsewhere, these lesions were classified further by degree of cytological atypia. ${ }^{22}$ The pattern and degree of the hyperplastic changes were graded separately, and an atypia score was derived by summing these two scores. The proliferative disorders which occurred most frequently were those displaying adenotic or epitheliotic changes. Epitheliosis with the highest atypia score is equivalent to atypical lobular hyperplasia described by Page et al, ${ }^{25}$ and to the lesion described by Black and Kwon ${ }^{27}$ as atypia grade 3-5 of the terminal ductal-lobular unit. The most severely atypical form of adenosis is equivalent to atypical ductal hyperplasia described by Page et al. ${ }^{28}$ Subjects found to have non-proliferative forms of benign breast disease or uncomplicated cystic disease formed the biopsy control group.

\section{DATA COLLECTION}

Subjects were interviewed in their homes by trained interviewers who administered questionnaires in a standardised manner. Cases and biopsy controls were interviewed as soon as possible after diagnosis; for cases, the average interval between diagnosis and interview was 2.8 months, and for biopsy controls, 2.9 months. Each community control was interviewed as soon as possible after her corresponding case had been enrolled in the study, and the average interval was 1.3 months.

Sociodemographic and medical information was collected from the study subjects with an interviewer administered questionnaire. A self administered questionnaire addressed dietary habits, alcohol consumption, and smoking history. Subjects were asked to indicate their cigarette consumption (in cigarettes per day) at various ages, from which a measure of lifetime cigarette consumption (in cigarette-years) was obtained by summing the product of each level of consumption by the number of years that level of consumption was maintained.

\section{DATA ANALYSIS}

For comparisons of cases and community controls, conditional logistic regression ${ }^{29}$ was used to derive maximum likelihood estimates of relative risk (RR) and associated $95 \%$ confidence intervals $(\mathrm{CI})$ and to assess the statistical significance of the trend in risk of benign proliferative epithelial disorders across categories of exposure. For comparisons of cases and biopsy controls, unconditional logistic regression ${ }^{29}$ 
Table 1 Risk of benign proliferative epithelial disorders of the breast in the total study population, and in premenopausal and postmenopausal women, by history of cigarette smoking.

Relative risk (95\% CI)



* Reference category. Note: for the total study population and for premenopausal women, relative risks derived from comparison of cases with community controls are matched relative risks, and cannot be calculated directly from the unmatched distribution of cases and controls shown in the table.

† Results for current smokers are shown above. 
was used, with adjustment for age. Estimates of effect adjusted for menopausal status, income, number of medical examinations per year, frequency of practice of breast self examination, Quetelet's index, alcohol consumption, and ever use of oral contraceptives were similar to unadjusted estimates, and only the latter are presented here.

Since the effect of cigarette smoking on hormonal processes in women may vary by menopausal status, ${ }^{1130}$ analyses were conducted overall and within menopausal strata. Women were considered postmenopausal if they had not had a menstrual period within the last 12 months, or if they had had a bilateral oophorectomy. Women who had had a hysterectomy before cessation of menstruation and who retained at least one ovary were allotted to menopausal strata on the basis of age. Those who were aged 56 years or more (and thus were more than five years older than the median age at natural menopause in the community controls) were deemed to be postmenopausal; those aged 44 years or less were deemed premenopausal, and the remainder perimenopausal. When women whose menopausal status was estimated in this way were excluded from analyses within menopausal strata, the results were not substantially affected. Therefore only the results based on the larger strata are presented here. Since there were relatively few postmenopausal study subjects (47 cases, 53 community controls, 46 biopsy controls), analyses in this stratum were restricted to calculation of risk for those who had ever smoked, ex-smokers and current smokers, relative to that for those who had never smoked. Also, for comparisons of postmenopausal cases and community controls, matching was broken (since there were relatively few case-control pairs concordant on postmenopausal status) and analyses were performed using unconditional logistic regression, which may have biased the estimates of effect conservatively. ${ }^{29}$
In a final set of stratum specific analyses, risk of benign proliferative epithelial disease in association with a history of ever having smoked cigarettes was examined by degree of cytologic atypia. Log-linear models ${ }^{31}$ were used to assess whether there was a trend in the risk of benign proliferative epithelial disease across levels of atypia: with community controls as the comparison group, matching was broken, which may have introduced conservative bias into the test for trend.

\section{Results}

In the total study population, there was little evidence for an association between cigarette smoking and risk of benign proliferative epithelial disease (table 1). Risk for ever smokers differed little from that for never smokers, and when risk was examined by current smoking status, neither ex-smokers nor current smokers were at markedly altered risk. There was little variation in risk of benign proliferative epithelial disease with amount currently smoked, and there was no suggestion of trends in risk with cigarette-years of exposure, and with years since women had first and last smoked cigarettes (data not shown). Women who had first smoked cigarettes at 20 years of age or later were at reduced risk of benign proliferative epithelial disease, an association of borderline statistical significance when cases were compared with community controls (data not shown). Results of analyses performed after exclusion of women with a past history of an unbiopsied breast lump or with a biopsy showing fibroadenoma but no surrounding epithelial proliferation differed little from those based on the total study population, and are not presented here.

When risk was examined within menopausal strata, the patterns were mostly similar to those described above (table 1). The main exceptions were in

Table 2 Risk of benign proliferative epithelial disorders of the breast in association with a history of ever having smoked cigarettes, by degree of cytological atypia.

\begin{tabular}{|c|c|c|c|c|}
\hline \multirow[b]{2}{*}{ Study subjects } & \multirow[b]{2}{*}{$\begin{array}{l}\text { Ever } \\
\text { smoked }\end{array}$} & \multirow[b]{2}{*}{$\begin{array}{l}\text { Never } \\
\text { smoked }\end{array}$} & \multicolumn{2}{|c|}{ Relative risk $(95 \% \mathrm{CI})^{*}$} \\
\hline & & & $\begin{array}{l}\text { Cases } v \\
\text { community controls }\end{array}$ & $\begin{array}{l}\text { Cases } v \\
\text { biopsy controls }\end{array}$ \\
\hline \multicolumn{5}{|l|}{ Cases: } \\
\hline No atypia & 62 & 69 & $0.8(0.5-1.6)$ & $1.0(0.6-1.5)$ \\
\hline Mild atypia & 59 & 81 & $0.8(0.5-1.3)$ & $0.8(0.5-1.3)$ \\
\hline Moderate atypia & 21 & 49 & $0.5(0 \cdot 2-1 \cdot 1)$ & $0.5(0.3-0.9)$ \\
\hline Severe atypia & 24 & 18 & $2.3(0.9-6.1)$ & $1.6(0 \cdot 8-3 \cdot 2)$ \\
\hline Community controlst & 181 & 202 & - & - \\
\hline Biopsy controlst & 89 & 103 & - & - \\
\hline
\end{tabular}

* Tests for linear trend: cases $v$ community controls, $\chi_{1}^{2}=1 \cdot 16, p=0 \cdot 28$; cases $v$ biopsy controls, $\chi_{1}^{2}=0.53, p=0.47$.

$\dagger$ Reference category. Note: relative risks derived from comparison of cases with community controls are matched relative risks, and cannot be calculated directly from the unmatched distribution of cases and controls shown in the table. 
premenopausal women: with community controls, there was a small decrease in risk with increasing cigarette-years of exposure, and a statistically significant reduction in risk of benign proliferative epithelial disease for women who had first smoked cigarettes at 20 years of age or later; and with biopsy controls, there was some suggestion of an increase in risk with cigarette-years of exposure. However, of the associated trends, only that for the age at which cigarettes were first smoked was statistically significant. After adjustment for potential confounding, the point estimates of effects were mostly similar to the unadjusted estimates, and none of the trends in risk were statistically significant (for age first smoked, $p=0 \cdot 106$ ).

Irrespective of the control group used, there was no trend in the risk of benign proliferative epithelial disease in association with a history of ever having smoked cigarettes, when this was examined by degree of cytological atypia (table 2). For both control groups, cigarette smoking was associated with reduced risk of mild and moderate degrees of atypia, and with increased risk of severe atypia. However, only one of the point estimates (for cases with a moderate degree of atypia versus biopsy controls) was statistically significant.

\section{Discussion}

The association between cigarette smoking and risk of benign breast disease has been examined previously in three case-control, ${ }^{161719}$ two cohort, ${ }^{1820}$ and one cross sectional study. ${ }^{21}$ Of the case-control studies, one showed the risk of cystic disease in ever smokers to be $60 \%$ higher than in never smokers, ${ }^{16}$ while the other two ${ }^{17}{ }^{19}$ showed ever smokers, and especially current smokers, ${ }^{19}$ to have reduced risk of fibrocystic disease and fibroadenoma. Cigarette smoking was not related to risk of benign breast disease in one of the cohort studies, ${ }^{20}$ and was weakly inversely related to fibroadenoma in the other. ${ }^{18}$ In the cross sectional study, cigarette smoking was inversely related to fibrocystic disease. ${ }^{21}$

Benign breast disease diplays considerable histological diversity, and it is possible that the various subgroups are related differently to cigarette smoking. In this study, cases were women with epithelial proliferation, and the results provide little support for an effect in this subgroup: overall, and within menopausal strata, risk of benign proliferative epithelial disease for women who had ever smoked, and risk for ex- and current smokers, was mostly similar to that for women who had never smoked.

The results of the present study, and indeed those of earlier studies, may have been influenced by the several sources of bias to which studies of benign breast disease seem predisposed. Since an unknown proportion of women with benign breast disease comes to clinical attention, and since, of those who do, an unknown proportion proceeds to biopsy, studies of biopsy confirmed benign breast disease are generally based on an unknown proportion of all women with the disease. Therefore the target population for any generalisations which can be made from most studies of benign breast disease is limited to women with biopsy determined disease. In the present study, in an attempt to reduce the inherent detection and selection biases, two control groups were selected: community controls, who were matched to cases on age and socioeconomic grading of area of residence, and biopsy controls, who comprised an unmatched group ascertained from the same source as the cases. In previous studies, community controls ${ }^{16}$ and hospital controls ${ }^{17}{ }^{19}$ have been selected. Use of each of these control groups can be criticised: community controls may be inappropriate since the case series may not represent all cases; ${ }^{19}$ hospital controls may be inappropriate since smokers may be overrepresented amongst them; and controls who have undergone the same diagnostic procedure as the cases may be inappropriate as their use may result in $\mathbb{D}$ "overmatching". ${ }^{32}$ However, it has been shown that when the possibility of serious underdiagnosis exists, then, in the absence of estimates of exposure specific detection rates and exposure specific rates of special treatment (eg, biopsy), it is not possible to determine a priori which control group will give the correct results. ${ }^{33}$

Misclassification of study subjects with respect to exposure and disease status could also have influenced the results of studies to date. The former is a problem in most epidemiological studies, but there is little reason to suspect differential misclassification of exposure in any of the studies of cigarette smoking and benign breast disease; non-differential misclassification of exposure biases estimates of effect conservatively. ${ }^{34}$ With respect to misclassification of disease status, it is possible that some non-cases have had undetected benign breast disease. In the present study, it was possible to address this issue to a limited extent by excluding women with a history of an unbiopsied breast lump; after excluding these women, the results were largely unchanged. In general, the consequence of this type of misclassification will be to bias estimates of effect conservatively.

Similar proportions of cases $(75.6 \%)$ and biopsy controls $(74.1 \%)$ were included in the study, while the response rate in the community controls $(65.8 \%)$ was somewhat lower. Differential non-response could have distorted the results presented here, although they were similar irrespective of the control group used for comparative purposes. 
When viewed in aggregate, the results of studies of cigarette smoking and benign breast disease are conflicting. Among possible explanations for the discrepant results are chance, and the effects of the biases noted above. Also, it is possible that the effect of smoking is confined to one particular histological subgroup, and that there have been differences between studies in the distribution of cases by histology. Finally, an indirect protective effect of cigarette smoking might be counterbalanced by a direct proliferative influence mediated by constituents of cigarette smoke (which have been found in breast fluid aspirates ${ }^{35}$ ). Given these considerations, the possibility of an inverse association between cigarette smoking and risk of benign breast disease cannot be excluded. Therefore there is a need for further cohort studies, and for case-control studies in which more than one control group is employed. Studies of populations surveyed for breast changes would be particularly useful.

This work was supported by grants from the Section 16 Committee of the South Australian Health Commission and from the Education or Research Appeal Fund of the Royal College of Pathologists of Australia. We thank Dr N G Carter for permission to use the laboratory facilities at Gribbles; Mr R Pearce for administrative help; Mr S G Stevens for the histological preparations; Ms C Powell, Mrs E Raymond, Mrs P Rogers and Ms M Juras for assistance with interviewing of the study subjects; and Mrs S Record for assistance with computing.

Address for correspondence and reprints: Dr T E Rohan, NCIC Epidemiology Unit, Faculty of Medicine, McMurrich Building, University of Toronto, Toronto, Ontario M5S 1A8, Canada.

\section{References}

${ }^{1}$ Cole P, Elwood JM, Kaplan SD. Incidence rates and risk factors of benign breast neoplasms. Am J Epidemiol 1978; 108: 112-20.

2 Preece PE, Hughes LE, Mansel RE, Baum M, Bolton PM, Gravelle IH. Clinical syndromes of mastalgia. Lancet 1986; ii: 670-3.

${ }^{3}$ Trapido EJ, Brinton LA, Schairer C, Hoover R. Estrogen replacement therapy and benign breast disease. $J$ Natl Cancer Inst 1984; 73: 1101-5.

${ }^{4}$ Berkowitz GS, Kelsey JL, Holford TL, et al. Estrogen replacement therapy and fibrocystic disease in postmenopausal women. Am J Epidemiol 1985; 121: 238-45.

5 Jick SS, Walker AM, Jick H. Conjugated estrogens and fibrocystic breast disease. Am J Epidemiol 1986; 124: 746-51

${ }^{6}$ Ernster VL. The epidemiology of benign breast disease. Epidemiol Rev 1981; 3: 184-202.
${ }^{7}$ Ernster VL, Wrensch MR, Petrakis NL, et al. Benign and malignant breast disease: initial results of serum and breast fluid analyses of endogenous estrogens. J Natl Cancer Inst 1987; 79: 949-60.

${ }^{8}$ Reed MJ, Cheng RW, Noel CT, Dudley HAF, James VHT. Plasma levels of estrone, estrone sulfate, and estradiol and the percentage of unbound estradiol in postmenopausal women with and without breast disease. Cancer Res 1983; 43: 3940-3.

${ }^{9}$ England PC, Skinner LG, Cottrell KM, Selwood RA. Serum oestradiol-17 $\beta$ in women with benign and malignant breast disease. Br J Cancer 1974; 30: 571-6.

${ }^{10}$ Sitruk-Ware R, Sterkers N, Mauvais-Jarvis P. Benign breast disease I: hormonal investigation. Obstet Gynecol 1979; 53: 457-60.

11 Baron JA. Smoking and estrogen-related disease. Am J Epidemiol 1984; 119: 9-22.

12 Ross RK, Pike MC, Vessey MP, Bull D, Yeates D, Casagrande JT. Risk factors for uterine fibroids: reduced risk associated with oral contraceptives. $\mathrm{Br}$ Med J 1986; 293: 359-62.

${ }^{13}$ Cramer DW, Wilson E, Stillman RJ, et al. The relation of endometriosis to menstrual characteristics, smoking and exercise. JAMA 1986; 255: 1904-8.

${ }^{14}$ Khaw K-T, Tazuke S, Barrett-Connor E. Cigarette smoking and levels of adrenal androgens in postmenopausal women. $N$ Engl $J$ Med 1988; 318: 1705-9.

${ }^{15}$ Michnovicz JJ, Hershcopf RJ, Naganuma H, Bradlow HL, Fishman J. Increased 2-hydroxylation of estradiol as a possible mechanism for the anti-estrogenic effect of cigarette smoking. $N$ Engl J Med 1986; 315: 1305-9.

${ }^{16}$ Nomura A, Comstock GW, Tonascia JA. Epidemiologic characteristics of benign breast disease. Am J Epidemiol 1977; 105: 505-12.

${ }^{17}$ Pastides H, Najjar MA, Kelsey JL. Estrogen replacement therapy and fibrocystic breast disease. Am J Prev Med 1987; 3: 282-6.

${ }^{18}$ Ramcharan S, Pellegrin FA, Ray RM, Hsu JP. Walnut Creek contraceptive drug study: a prospective study of the side effects of oral contraceptives. III. Bethesda: National Institutes of Health, 1981.

${ }^{19}$ Berkowitz GS, Canny PF, Vivolsi VA, Merino MJ, O'Connor TZ, Kelsey JL. Cigarette smoking and benign breast disease. J Epidemiol Community Health 1985; 39: 308-13.

${ }^{20}$ Mant D, Vessey MP, Smith MA, Yeates D. Cigarette smoking and benign breast disease. $J$ Epidemiol Community Health 1986; 40: 277-8.

${ }^{21}$ Wyshak G, Frisch RE, Albright NL, Albright TE, Schiff I. Cigarette smoking and benign breast disease (Letter to the Editor). N Engl J Med 1988; 319: 1736-7.

22 Rohan TE, Cook MG. Alcohol consumption and risk of benign proliferative epithelial disorders of the breast in women. Int J Cancer 1989; 43: 631-6.

${ }^{23}$ Stimson RJ, Cleland EA. A socio-economic atlas of Adelaide. An analysis of the 1971 Census. Adelaide: School of Social Sciences (Flinders University of South Australia) and Department of Community Welfare, 1975.

${ }^{24}$ Bonser GM, Dossett JA, Jull JW. Human and experimental breast cancer. London: Pitman Medical, 1961.

${ }^{25}$ Page DL, Zwag RV, Rogers LW, Williams LT, Walker WE, Hartmann WH. Relation between component parts of fibrocystic disease complex and breast cancer. $J$ Natl Cancer Inst 1978; 61: 1055-63.

${ }^{26}$ Azzopardi JG. Problems in breast pathology. Vol II in Series, Major problems in pathology. London: WB Saunders, 1979. 
${ }^{27}$ Black MM, Kwon S. Precancerous mastopathie: structural and biological considerations. Path Res Pract 1980; 166: 491-514.

${ }_{28}$ Page DD, Dupont WD, Rogers LW, Rados MS. Atypical hyperplastic lesions of the female breast. A long-term follow-up study. Cancer 1985; 55: 2698-708.

${ }_{29}$ Breslow NE, Day NE. Statistical methods in cancer research. Vol. I. The analysis of case-control studies. Lyon: IARC Scientific Publications, 1980.

${ }^{30}$ Baron JA, Greenberg ER. Cigarette smoking and estrogen-related diseases in women, In: Rosenberg MI, ed. Smoking and reproductive health. Littleton (Mass): PSG, 1987: 149-60.
${ }^{31}$ Nelder JA. Log Linear models for contingency tables: a generalization of classical least squares. Appl Statist 1974; 23: 323-9.

32 Vessey MP. Comment. J Chron Dis 1979; 32: $64-6$.

${ }^{33}$ Greenland S, Neutra R. An analysis of detection bias and proposed corrections in the study of estrogens and endometrical cancer. J Chron Dis 1981; 34: 433-8.

${ }^{34}$ Blettner M, Wahrendorf $J$. What does an observed relative risk convey about possible misclassification? Methods Inf Med 1984; 23: 37-40.

${ }^{35}$ Petrakis NL, Gruenke LD, Beelen TC, Craig JC, Castagnoli $\mathbf{N}$. Nicotine in breast fluid of non-lactating women. Science 1978; 199: 303-5. 